\title{
Immer mehr Geld für Gesundheit? Immer mehr Gesundheit fürs Geld!
}

\author{
Jürg Schlup \\ Dr. med., Präsident der FMH
}

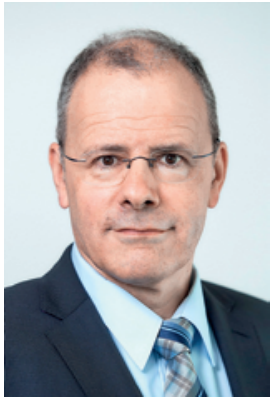

«Kostenexplosion noch nicht im Griff - Fünfprozentiger Prämienanstieg in der Krankenversicherung" [1] titelte die NZZ bereits vor 20 Jahren und berichtete, nach Ausfassung des Bundesrats müsse die «Mengenausweitung namentlich im ambulanten Bereich" gebremst werden. Auch die diesjährige Prämiendiskussion lässt wieder viele Berichte erwarten, die zu belegen scheinen, dass unser Gesundheitswesen unerschwinglich wird. Auf der Basis der so geschürten Ängste lassen sich dann (zu) simple Lösungsangebote einfach proklamieren: aktuell liegen als Heilsversprechen besonders Kostendeckel und staatliche Steuerung im Trend. Obwohl die «Kosten-

\section{Nach 50 Jahren «Kostenexplosion» ist die} Zahlungsunfähigkeit ausgeblieben - und der Wohlstand gewachsen.

explosion" im Gesundheitswesen - die in der NZZ vor knapp 50 Jahren erstmals erwähnt wurde [2] - uns nach einem halben Jahrhundert noch nicht in die Zahlungsunfähigkeit getrieben hat, funktioniert sie als Schlagwort nach wie vor fantastisch.

Referenzen

1 NZZ, 4./5.10.1997; Nr. 230, S.13. Kostenexplosion noch nicht im Griff. Fünfprozentiger Prämienanstieg in der Krankenversicherung.

NZZ, 10.7.1968; Morgenausgabe Nr. 417, S.9. Gesundheitswesen und Krankenversicherung. Ein Diskussionsbeitrag.

3 Früh, Gyger, Reich. Helsana-Report. Ausgabenentwicklungen in der Gesundheitsversorgung. Dezember 2016.

4 NZZ, 16.9.1986; Nr.214, S.63. Leserbrief Prof. R. Tscholl, «Kostenexplo sion» im Gesundheitswesen.

5 Telser, Fischer, Leukert, Vaterlaus. Gesundheitsausgaben und Krankheitskosten. Interpharma/Polynomics, September 2011
Da sich die ständig wiederholten Halbwahrheiten für viele Bürger beim Blick ins Portemonnaie zu bestätigen scheinen, finden deutlich differenziertere Betrachtungen der Kostenentwicklung leider kaum Gehör: "Als Volkswirtschaft kann die Schweiz die steigenden Gesundheitskosten gut verkraften" [3] schreibt die Helsana, weil nämlich «trotz wachsendem Anteil der Gesundheitskosten am BIP der um die Gesundheits-

kosten bereinigte Wohlstand um ein Drittel gestiegen ist» [3]. Wäre der Anstieg der monatlichen Durchschnittsprämie zwischen 2006 und 2014 um insgesamt 100 Franken eine Prämienexplosion, hätten wir gleichzeitig eine ungleich grössere Konsumexplosion (+325 CHF), Steuerexplosion (+370 CHF) und Ersparnisexplosion (+715 CHF) zu verzeichnen [3]. Dass viele Haushalte von diesen nationalen Durchschnittswerten deutlich abweichen, sollte uns für das Problem der Lastenverteilung auf die verschiedenen Bevölkerungsgruppen sensibilisieren, statt die Ausgabenentwicklung zum «Sündenbock für Verteilungsprobleme» zu machen [3].
Im reichsten Land der Welt Ausgaben zu deckeln und damit Leistungen zu rationieren, hilft einkommensschwächeren Haushalten nämlich nicht: In der zwangsläufig folgenden Zwei-Klassen-Medizin kämen sie als erste unter die Räder.

Der Begriff der «Kostenexplosion» taugt aber auch deshalb nichts, weil er die "Nutzenexplosion" ausblendet, die der Ausgabenentwicklung gegenübersteht. Auch dies war bereits vor über 30 Jahren sehr anschaulich nachzulesen: «Wenn ein Kunde gestern für einen Cervelat Fr. 1.- bezahlte und heute für denselben Kauf Fr. 50.hinlegen müsste, so würde er vor einer echten Kostenexplosion stehen. Wenn der gleiche Kunde aber heute statt eines Cervelats $1 \mathrm{~kg}$ Rindfleisch zu Fr. 50.-kauft, so erleidet er keine Kostenexplosion, sondern er verursacht eine Ausgabenexplosion, weil er nicht mehr Cervelat, sondern Filetfleisch konsumieren will» [4]. Ähnlich erbringt das Gesundheitswesen für das zusätzlich aufgewendete Geld auch zusätzliche Leistungen - und Nutzen, weil mehr und mehr Patienten von immer besseren Diagnose- und Therapieverfahren profitieren. Die empirische Evidenz zeigt, "dass die höheren Gesundheitsausgaben der letzten dreissig Jahre durch den Nutzen [...] mehr als kompensiert wurden, die Krankheitskosten insgesamt also eher abgenommen haben", [5] wenn man neben den alljährlich heiss diskutierten direkten medizini-

\section{Der Ausgabenexplosion steht eine beachtliche} Nutzenexplosion gegenüber.

schen Kosten auch Produktivitätsverluste am Arbeitsplatz, die Pflege durch Angehörige etc. einbezieht. Eine gesellschaftliche Diskussion, ob wir uns Filet für alle leisten können - oder doch nur Cervelat - setzt eine Vorstellung vom Nutzen der «Filetversorgung» voraus und das Wissen, dass ihr Nutzen die Gesundheitsausgaben um bis zu 100 Prozent übersteigt [5]. Um diesen Nutzen zu illustrieren, wird eine neue Artikelserie nach einem ersten einleitenden Beitrag in dieser SÄZ allen Fachrichtungen die Möglichkeit geben, den Nutzen von Fortschritten in ihrer Disziplin aufzuzeigen. 